\title{
Image Compression Based on Centipede Model *
}

\author{
Binnur Kurt ${ }^{1}$, Muhittin Gökmen ${ }^{1}$, Anil K. Jain ${ }^{2}$ \\ 1 Istanbul Technical University, \\ Faculty of Elecrical and Electronics, Department of Computer Science, \\ Maslak, ISTANBUL 80626 - TURKEY \\ kurt@cs.itu.edu.tr, gokmen@cs.itu.edu.tr \\ 2 Michigan State University, Department of Computer Science, \\ East Lansing, MICHIGAN 48824 - U.S.A. \\ jain@cps.msu.edu
}

\begin{abstract}
We present an efficient contour based image coding scheme based on Centipede Model. Unlike previous contour based models which presents discontinuities with various scales as a step edge of constant scale, the centipede model allows us to utilize the actual scales of discontinuities as well as location and contrast across them. The use of the actual scale of edges together with other properties enables us to reconstruct a better replica of the original image as compared to the algorithm lacking this feature. In this model, there is a centipede for each edge segment which lies along the segment and the gray level variation across an edge point is represented by the difference between footholds and distance between left and right feet of the centipede. We obtain edges by using the recently introduced Generalized Edge Detector (GED) [1] which controls the scale and shape of the filter, providing edges suitable to the application in hand. The detected edge segments are ranked based on the weighted sum of the length of the segment, mean contrast and standard deviation of gray values on the segment. In our scheme, the compression ratio is controlled by retaining the most significant segments and by adjusting the distance between the successive foot pairs. The original image is reconstructed from this sparse information by minimizing a hybrid energy functional which spans a space called $\lambda \tau$-space. Since the GED filters are derived from this energy functional, we utilized the same process for detecting the edges and reconstructing the surface from them. The proposed model and the algorithm have been tested on both real and synthetic images. Compression ratio reaches to 180:1 for synthetic images while it ranges from 25:1 to 100:1 for real images. We have experimentally shown that the proposed model preserves perceptually important features even at the high compression ratios.
\end{abstract}

\section{INTRODUCTION}

The model based image compression has been attracted great interest as an attempt to exceed the boundaries of classical coding methods such as predictive

* This study is partially supported by TUBITAK grant EEEAG-154 and NSF grant INT-9500652. 
coding, information theory based methods, transform coding and vector quantization. One general image model often used characterizes the image in terms of contours and regions surrounded by them. In this framework, one class of algorithm requires an accurate partitioning of image into homogeneous closed regions $[2,3]$ (region based), whereas the other class of algorithms attempt to reconstruct the image from edge segments (edge based) and their neighborhood [2]. The former approach utilizes the uniformity of the each segmented region while the latter utilizes the differences between two regions. Even though much of the research efforts devoted to the region based approaches, it is more practical and efficient to extract edges by using high performance edge detectors instead of costly region segmentation. Gökmen et al. [4] developed a model based coding of fingerprint images based on ridges and valleys. Acar and Gökmen [5] utilized the weak membrane model of image in an attempt to carry out the edge detection and surface reconstruction by using the same process. This unification enables to use the same minimization process for both edge detection and surface reconstruction, and simplifies the coding scheme. However, the reconstructed images look somewhat artificial due to treating each edge as a step function by disregarding its actual scale. As known, the boundaries of physical structures in the world give rise blurred transitions in image intensities corrupted by noise instead of step discontinuities. Elder and Zucker [6] suggested a method to estimate the scale of edge by modeling it as a step function blurred by a Gaussian kernel.

In the following section, we introduce a coding scheme which combines all these features by utilizing a novel model called centipede model. In section 3 , we describe how to encode the model parameters, together with ranking the edge segments and modeling the gray level variation along a segment by fitting polynomial curves. Also included in this section is the reconstruction of original image from encoded model parameters by minimizing a hybrid energy functional. In the last section, the performance of the algorithm on synthetic and real images are quantitatively and qualitatively analyzed.

\section{CENTIPEDE MODEL}

In our coding scheme, each connected edge segment as well as intensity variations around segment is described by a model called Centipede Model. A centipede, shown in Fig. 1, consists of a backbone along the edge segment and a number of legs approximately parallel to each other and normal to the backbone at the intersection points. Our centipede model consists of six parameters. When a centipede is placed over an edge segment, the transition of gray values can be captured by these parameters. Representing the position of a centipede parametrically by $V(s)=(x(s), y(s))$, the model can be described by the pentuple $\left(V(s), I(s), C_{L}(s), C_{R}(s), W_{L}(s), W_{R}(s)\right)$ defined as

$$
\begin{array}{r}
C_{L}(s)=I\left(V_{L}(s)\right)-I(V(s)), \quad C_{R}(s)=I\left(V_{R}(s)\right)-I(V(s)) \\
W_{L}(s)=\left|V(s)-\inf _{V_{L}}\left\{\left|V-V_{L}\right|:\left|\frac{\partial I(x, y)}{\partial n}\right|_{V=V_{L}}<T_{L}\right\}\right|
\end{array}
$$




$$
W_{R}(s)=\left|V(s)-\inf _{V_{R}}\left\{\left|V-V_{R}\right|:\left|\frac{\partial I(x, y)}{\partial n}\right| V=V_{R}<T_{R}\right\}\right|
$$

where $I(x, y)$ is the image intensity at $(\mathrm{x}, \mathrm{y}), \frac{\partial I(x, y)}{\partial n}=n . \nabla I$ is intensity gradient in the direction $n$, normal to $V(s), T_{L}$ and $T_{R}$ are very small threshold values for the intensity gradients.

Thus, the model consists of geometry of edge segment $V(s)$, intensity value on edge $I(s)$, the lengths of left and right legs $W_{L}$ and $W_{R}$ capturing the scale of the edge, and left and right footholds, $C_{L}$ and $C_{R}$ corresponding to the contrast across this edge, respectively. The length of a $\operatorname{leg}, W_{L}$, corresponding to the scale of an edge, is determined as the distance from this edge point to the closest point for which the gradient magnitude along the direction normal to the edge direction is less than a predetermined threshold value $T_{L}$. The entire image is modeled as a family of centipedes placed on edge segments as shown in In Fig. 1. The image

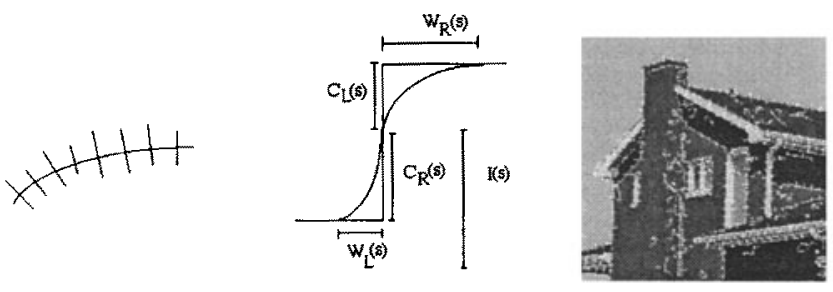

Fig. 1. Centipede Model, Parameters, Centipede Model overlied on the House image (From left to right)

is compressed by efficiently encoding the parameters of the centipede, and the original image is formed by reconstructing a surface from these parameters by minimizing a hybrid energy functional.

\section{ENCODING AND DECODING IMAGES}

The first part of the algorithm is to detect the edges by using the Generalized Edge Detector which encompasses most of the widely used edge detectors such as Canny's, Deriche's, Sarkar and Boyer's, Shen and Kastan's edge detectors. Edges are then traced to detect the distinct contours. These contours are coded by differential chain coding. Edge following algorithm forces the edge segments being as smooth as possible so that the gain of the compression of binary image with differential chain code followed by Huffman coding is maximized.

The second part of the compression algorithm is to select the perceptually most important edge segments among these contours obtained by tracing the edge segments. This is achieved by first assigning a priority to each edge segment by simply calculating the weighted sum of normalized set of contour length, average contrast along normal direction and average curvature evaluated directly 
from the differential chain code representation of the curve. Priority assigned to the contour $C_{i}$ is given by

$$
\text { Priority }\left(C_{i}\right)=w_{l} \cdot \text { Length }\left(C_{i}\right)+w_{c} \cdot \text { Contrast }\left(C_{i}\right)+w_{u} \cdot \text { Curvature }\left(C_{i}\right) .
$$

In the previous section, a method is introduced to extract the model parameters. The parameters along a segment are modeled by polynomials, and coefficients of each polynomial are coded. At the decoding part, decoder constructs two images, a binary edge map and intensity image obtained from model parameters which can be constructed simply by evaluating the polynomial at each edge point. Since both images are sparse, we use hybrid energy functional to span a surface through these points. For this purpose, the surface reconstruction problem is set as finding a function $f(x, y)$ which minimizes

$$
\begin{gathered}
E(f ; \lambda, \tau)=\int_{\Omega} \int \beta(x, y)(f(x, y)-d(x, y))^{2}+\lambda(1-\tau)\left(f_{x}^{2}(x, y)+f_{y}^{2}(x, y)\right) \\
+\lambda \tau\left(f_{x x}^{2}(x, y)+2 f_{x y}^{2}(x, y)+f_{y y}^{2}(x, y)\right) d x d y
\end{gathered}
$$

where $\lambda$ controls the smoothness of the surface and $\tau$ controls the continuity of the surface. Properties of the hybrid model is explained in [1]. Minimization of functional given by ( 3 ) is obtained iteratively by Successive Over-Relaxation. In order to eliminate a possible blurring across discontinuities, we defined the centipede foots, i.e. $C_{L}$ and $C_{R}$, as crease points and vanish the last term in (3) including the second derivatives at these points. As described in [1], the Generalized Edge Detector is derived from the functional in (3), the scale and the shape of the GED is controlled by the $\lambda$ and $\tau$ parameters in the hybrid functional. Thus we utilize the same process for both detecting edges and reconstructing images from them, unlike unrelated processes such as Canny edge detection and surface interpolation.

\section{RESULTS AND CONCLUSIONS}

The proposed model-based image coding has been applied to various synthetic and real images. We first consider the effect of ranking the edge segments and selecting only most significant segments on the reconstructed image quality. Fig.2(a) shows the original Lenna and House images. Fig.2(b,c,d,e) show the selected edges from a complete edge map obtained by the GED (with $\lambda=0.5$ and $\tau=0.5$ ). From these edge maps, $100 \%, 75 \%, 50 \%$ and $25 \%$ of edges are retained and these edges together with the reconstructed images are shown in Fig.2(b),(c),(d) and (e), respectively. The quantitative results for this test are also shown in this figure. The qualitative and quantitative evaluation of results indicates that the selection scheme works quite successfully. Even if very large portion of the edge segments are removed, the reconstructed image still contains most of the perceptually pertinent features. When we code the centipede parameters, we divided the each edge segment into blocks and approximate the parameters $I(s), C_{L}(s), C_{R}(s), W_{L}(s)$, and $W_{R}(s)$ over a block by fitting curves 
of order $n$. Thus in our coding scheme, the compression ratio and the quality of reconstructed image can be controlled by the the percentage of selected edge segments, the block size and the orders of approximations for the intensity, the contrast, and the edge width over the block.

Fig. 3 shows the detected edges, V(s), the intensities and estimated scales of edges and the centipede model superimposed on the original House and Lenna images in Fig.2(a). To reveal the coding performance of the proposed model, we considered various synthtetic and real images. For the synthetic checkerboard and bar images[5], compression ratios of $127: 1$ and 157:1 are achieved, respectively. Fig. 4 shows the original image, edge map and reconstructed images, from left to right, for House and Cameraman images. Compression ratios are $44: 1$ and $29: 1$, respectively. Fig. 5 shows how the reconstructed image is degraded as the compression ratio increases. As seen from these results, relatively high compression ratios can be achieved by the proposed scheme. One of the advantageous of this contour based approach as compared to transform based coding is that this scheme does not cause excessively blurred image or blocking artifacts as the compression ratio increases. It retains the most important features even for the very high compression ratios.

In conclusion, we presented a new model for contour based image compression. This model enables us to utilize the scale, brightness and contrast of edges. We developed a ranking scheme for edge segments so that the most significant edge segments can be kept after the removal of the edge segments to increase the compression ratio. We utilized an efficient way of encoding the model parameters by means of curve fitting, differential chain coding and Huffman coding. We used the similar process controlled by the same parameters, $\lambda$ and $\tau$, for both detecting edges and reconstructing original image from edges. All these combined features make the proposed centipede model very attractive alternative to the existing model based schemes.

\section{References}

1. M. Gökmen, A. K. Jain, " $\lambda \tau$-space representation of images and generalized edge detector," to appear in IEEE Trans. on PAMI, June 1997.

2. M. Kunt, M. Benard, R. Leonardi, "Recent results in high compression image coding," IEEE Transactions on Circuits and Systems, Vol. CAS-34, No. 11, pp. 1306-1336, November 1987.

3. H. Sanderson, G. Crebbin, "Region-based image coding using polynomial intensity functions," IEE Proc. on Vision and Image Proc., No.1, pp.15-23, Feb. 1996.

4. M. Gökmen, I. Ersoy, A. K. Jain, "Compression of fingerprint images using hybrid image model," Proc. IEEE Int'l Conf. On Image Proc., Vol.III, pp. 395-398, 1996.

5. T. Acar, M. Gökmen, "Image coding using weak membrane model," Proc. of Visual Comm. and Image Processing'94, pp. 1221-1230, Chicago, Mlinois, 1994.

6. J.H. Elder, S.W. Zucker, "Scale space localization, Blur and Contour based image coding," Proc. of Int. Conf. on Image Processing, pp. 1221-1230, 1996. 

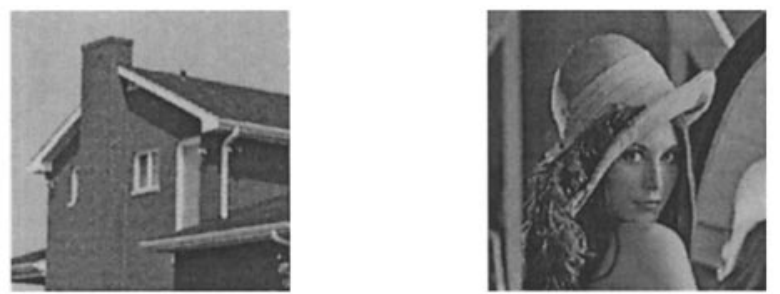

(a) Original House and Lenna Images
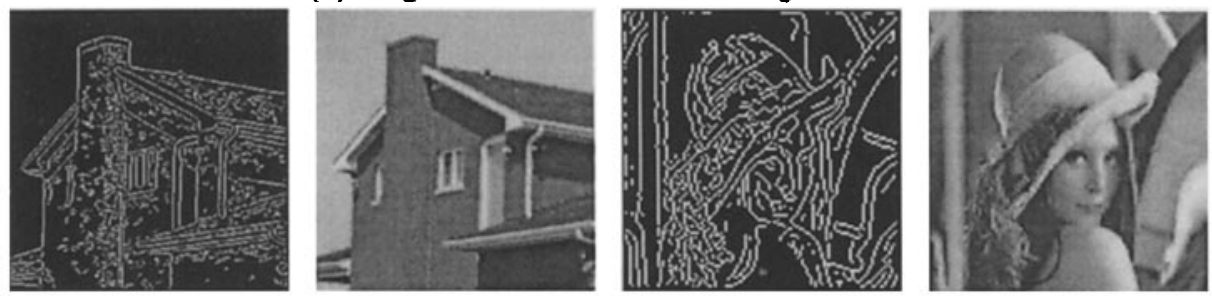

(b) $\% 100$ of edges are selected and $\mathrm{SNR}=(39.63,31.03)$
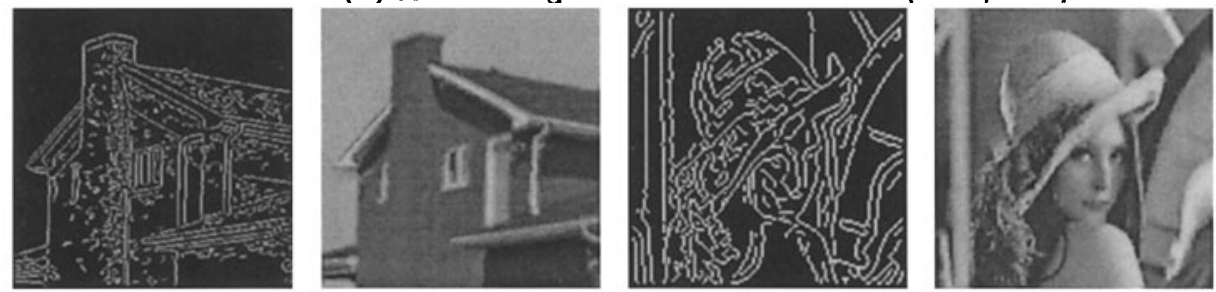

(c) $\% 75$ of edges are selected and $\mathrm{SNR}=(39.44,29.22)$
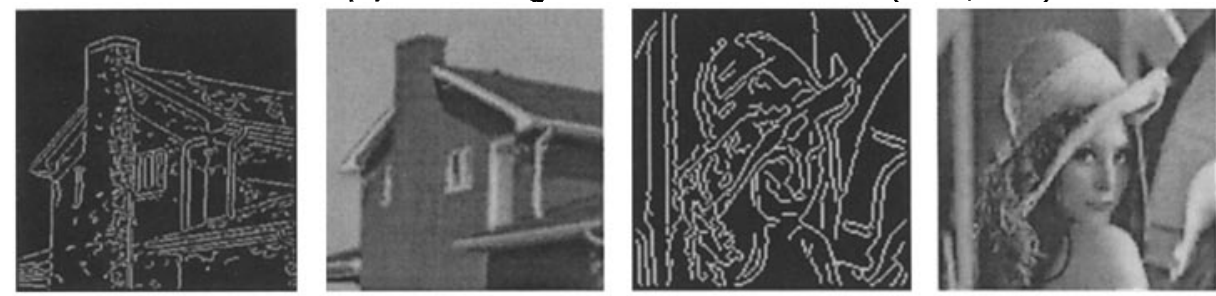

(d) $\% 50$ of edges are selected and $\mathrm{SNR}=(38.88,27.64)$
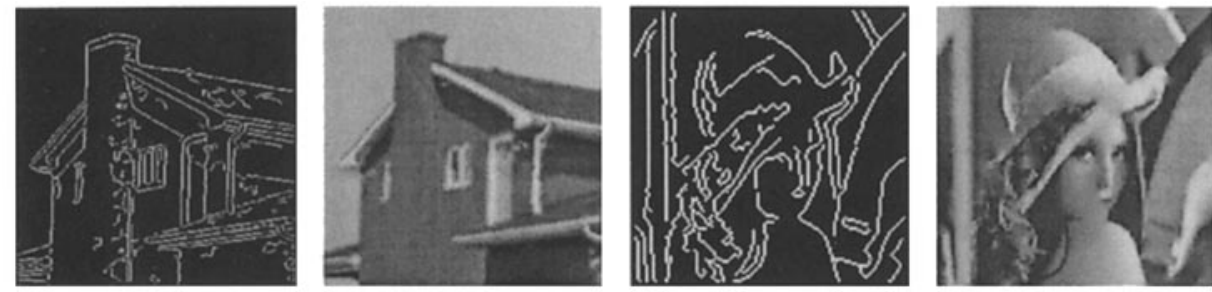

(e) $\% 25$ of edges are selected and $\mathrm{SNR}=(38.00,24.62)$

Fig. 2. The selected edges and reconstructed images for House and Lenna images and corresponding SNR(dB) values. From top to bottom, 100, 75, 50 and $25 \%$ of edges are retained. 


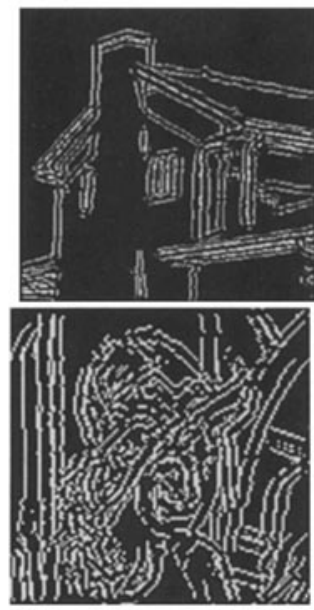

(a)
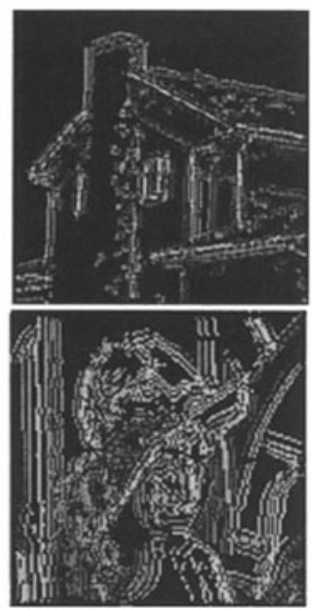

(b)

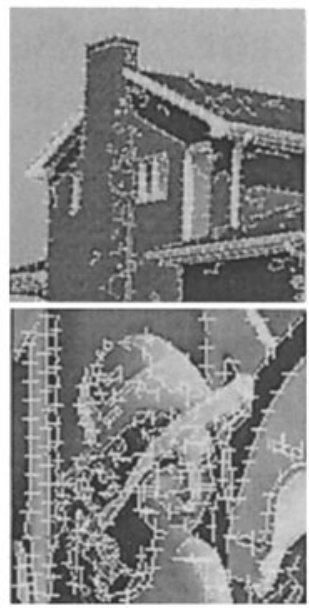

(c)

Fig. 3. Representation of detected edge scales.(a) Centipede Footholds, (b) Intensities on edges and footholds, (c) Centipede Model superimposed on the original House and Lenna images.
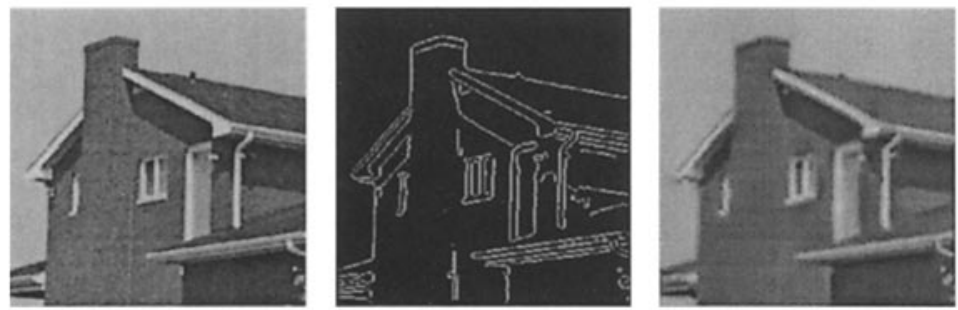

Compression Ratio : $44: 1$
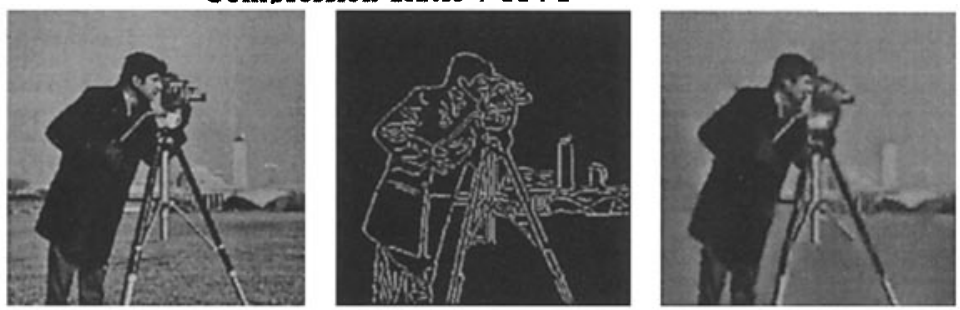

Compression Ratio : $29: 1$

Fig. 4. Original image, Edge Map and Reconstructed image for House and Cameraman images. 


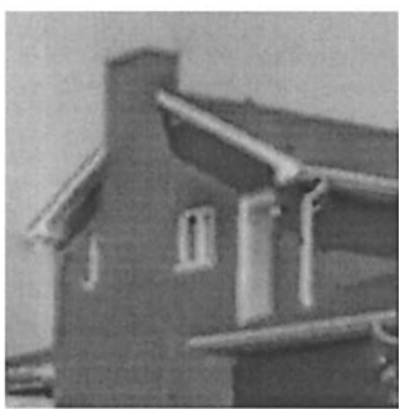

$\mathrm{CR}=37: 1$

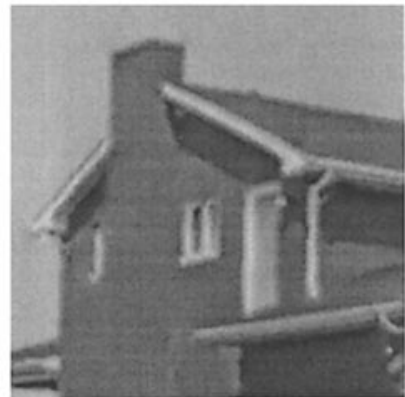

$\mathrm{CR}=48: 1$

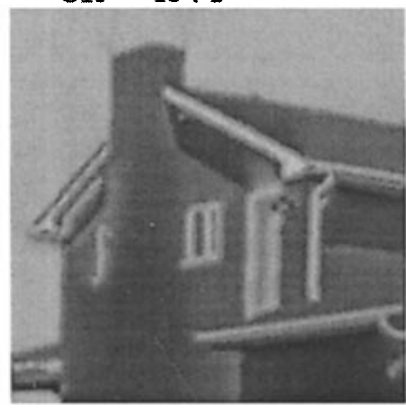

$\mathrm{CR}=74: 1$

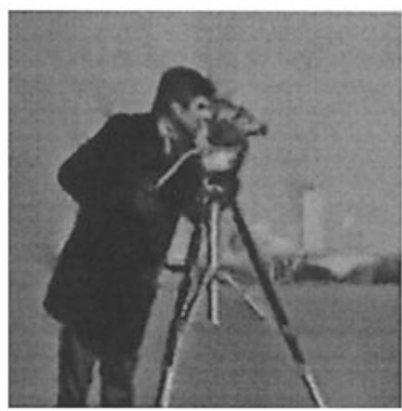

$\mathrm{CR}=22: 1$

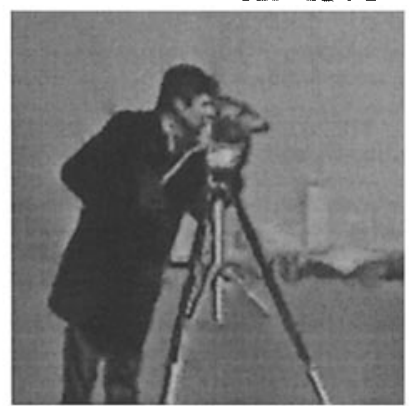

$\mathrm{CR}=29: 1$

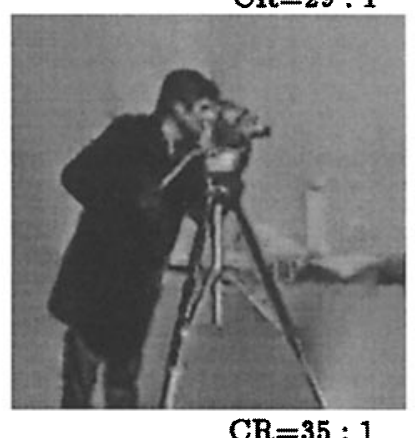

$\mathrm{CR}=35: 1$

Fig. 5. The reconstructed House and Cameraman images for the specified Compression Ratios (CR). 